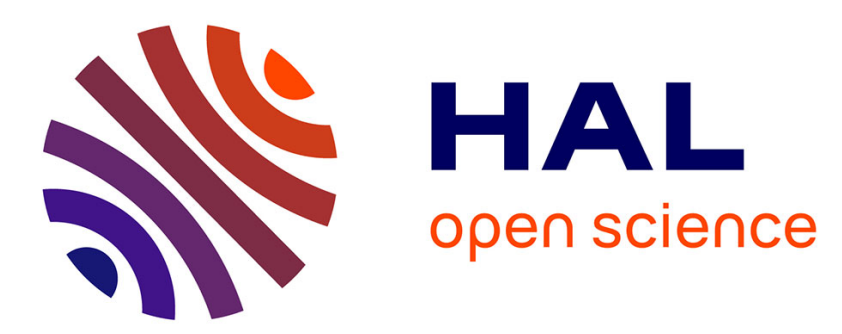

\title{
Application of Superconducting Electronics to Registration of Biomagnetic Signals
}

\author{
V. Sosnitsky, I. Vojtovich
}

\section{To cite this version:}

V. Sosnitsky, I. Vojtovich. Application of Superconducting Electronics to Registration of Biomagnetic Signals. Journal de Physique IV Proceedings, 1996, 06 (C3), pp.C3-417-C3-422. 10.1051/jp4:1996363 . jpa-00254281

\section{HAL Id: jpa-00254281 \\ https://hal.science/jpa-00254281}

Submitted on 1 Jan 1996

HAL is a multi-disciplinary open access archive for the deposit and dissemination of scientific research documents, whether they are published or not. The documents may come from teaching and research institutions in France or abroad, or from public or private research centers.
L'archive ouverte pluridisciplinaire HAL, est destinée au dépôt et à la diffusion de documents scientifiques de niveau recherche, publiés ou non, émanant des établissements d'enseignement et de recherche français ou étrangers, des laboratoires publics ou privés. 


\title{
Application of Superconducting Electronics to Registration of Biomagnetic Signals
}

\author{
V.N. Sosnitsky and I.D. Vojtovich \\ Institute of Cybernetics of National Academy of Sciences, 252022, Prospect Academica Glushkova 40, \\ Kiev, Ukraine
}

\begin{abstract}
Computer-aided systems used to measure superweak magnetic fields in a human body are created. A relaxation oscillation (RO) SQUID magnetometer is the basis of these systems. It is shown that if the RO operation mode is utilized, it becomes possible to improve the parameters and simplify the SQUID-magnetometer electronics. The software used for preprocessing provides the digital filtration, data input and accumulation, visual signalquality control and signal averaging and all this is done in a PC/AT. The magnetocardiogram (MCG) processing programs yield magnetic mapping, magnetic chart visualization, provide the correlational/ statistical analysis and magnetic signal source localization. The biomagnetic systems are now installed in the cardiological clinic. The spesialists examine MCGs, magnetoplethismograms and perfom magnetic liver biopsy. More then 400 patients have been examined and the experience gained after such examinations shows that biomagnetic investigations aimed at human body organ disease diagnosis are of high informational value.
\end{abstract}

\section{INTRODUCTION}

Vital activity of a living organism is accompanied by magnetic field radiation. Among its sources are ion movement, magnetic properties of materials participating in biological processes, ferromagnetic and paramagnetic particles penetrating into the organism from outside. These biomagnetic fields are very weak and their measurement became possible only after the sensors based on SQUIDs have been invented in which a record sensitivity to magnetic field - up to $10^{-15} \mathrm{~T}$ - is attained.

The measurement of biomagnetic fields is of the greatest interest from the standpoint of elaboration of new diagnosis methods in medicine, which is caused by the following advantages of magnetometry: contact-free measurements, including measurements through clothes or bandage, absolute safety and harmlessness; obtaining more reliable information about electric processes in organs (since the magnetic heterogeneity of body tissues is considerably lower than electric one); the possibility of measuring magnetic signals in any places and drowing spatial magnetic signals maps; high accuracy of localization of a magnetic signal source and a possibility of defining its configuration and distortions. Due to all these potentialities and advantages biomagnetometry is capable of giving such information about an organism which cannot be obtained using other methods. But realization of measurements with the help of supersensitive SQUID sensors is a difficult problem. It is connected with solving a number of tasks concerning noise immunity, filtration, multichannel input into computer, processing of signal collections, their representation, interpretation of measurement results, etc. We have created computer-aided systems in which all these and other problems are solved. One of the systems is used for recording of magnetic fields of the cardio-vascular system, the other - for investigation of magnetic susceptibility of various organs and blood. For measuring magnetic signals in computer-aided systems a RO SQUID magnetometer is developed which has a number of advantages in comparison to traditional designs. Each system has its own structural features and measurement methods. Signal processing and representation are performed by specially designed algorithms and programs. 


\section{RELAXATION OSCILLATION SQUID-MAGNETOMETER}

The traditional implementation of a SQUID-magnetometer is based on the relatively slow analog electronics which operates in the presence of microvolt-level signals. Such implementation possesses many drawbacks. The analog signal form requires a careful tuning-out from noises made by means of equipment shielding and signal filtering. Another approach consists in transition from the analog operation mode to the pulse one. And the unshunted tunnel Josephson junctions (JJ) with the hysteretic IVC are used here. For a signal to be measured in the pulse mode, the pulse of the voltage of a comparatively large amplitude of the gap voltage $V_{\mathrm{g}}$ order (millivolts) is used. The pulse mode creates the prerequisites for the device operation speed to be increased, for its dynamic range and functional possibilities to be extended, enhances the device noiseimmunity and simplifies the sensor manufacturing technology. The great pulse mode advantage consists in the fact that the SQUID characteristics are insensitive to the SQUID IVC deformations that emerge because of the spureous resonance occuring in an interferometer and an input spiral coil, between an interferometer and a coil, the interferometer branches/JJs asymmetry. All these factors are the reason for which the conventional SQUIDs characteristics fall down. In the traditional SQUIDs the MDM scheme with MF modulation is applied for the electronics $1 / \mathrm{f}$ noises to be reduced. The pulse operation mode does not require such modulation because the SQUID bias current is modulated.

The first variants of application of a pulse operation mode in the SQUID technology are presented in [1]. The SQUID is tested in this case under the constant frequency and with the sawtooth alternating current (AC). The external test AC source is also used in the SQUID Pulse Probability Modulation technology [2]. Since external AC sources are present in these papers, the readout electronics becomes more complicated and when multichannel systems are built the additional noises appear. In our opinion, this is the main reason why the test AC generation performed directly in a SQUID in the ROs form is the more promising approach.

We consider the application with respect to creation of the magnetometer of only such oscillations that emerge when a tunnel $\mathrm{JJ}$ of high capacity $\mathrm{C}$ is shunted by a resistance $\mathrm{R}$ and an inductance $\mathrm{L}$ that are connected serially. The bias current $I_{b}$, which meets the condition $I_{c}<I_{b}<V_{p} / R$, where $V_{p}=V_{c} \beta^{-1 / 2}, V_{c}=$ $I_{c} R_{n}$ - are the plasma and characteristic voltage of a JJ and $I_{c}, R_{n}$ - are the JJ critical current and JJ resistance, is supplied. The analysis shows [3] that if the condition $\tau \gg \tau_{n}$, where $\tau=L / R, \tau_{n}=C_{n}$, is met, then T, i.e. a RO period, is as follows:

$$
\left.T=T_{d}\left[1+(\pi / 2)\left(L_{c} L\right)\right]+(4 / \pi+\pi / 4) \tau_{w}, \quad T_{o}=\tau \ln /\left(1+I_{c} R /\left(V_{g}-R I_{b}\right)\right) /\left(1-I_{c} / I_{b}\right)\right], \quad L_{c}=\Phi_{d} / 2 \pi I_{c}
$$

From (1) we can see the dependence of SQUID critical current which, in its turn, depends upon the recorded MF. The authors suggested to use this principle for construction of magnetometers [4]. Our experience showed that good results could be obtained proceeding from the ROs of the relatively low frequencies (few $\mathrm{MHz}$ ), if the RO frequency (F) dependence on MF ( $\Phi)$ is used as the output signal. An operation point is selected in the sector of the greatest steepness $\mathrm{dF} / \mathrm{d} \Phi$. Under the negative feedback (NF) closure, an MF is strictly fixed in an interferometer ring and this leads to operation point fixation under the specified RO frequency. The similar approach later was described in [5] as well.

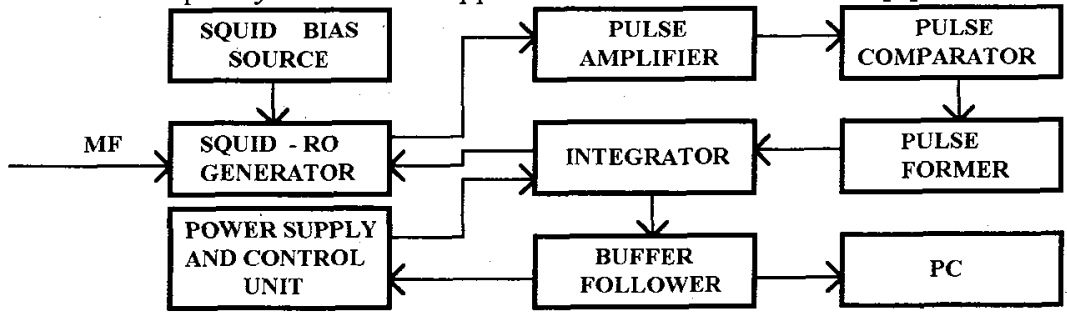

Figure 1: RO Magnetometer: A Flowchart. 
The RO generator based on the direct current (DC) SQUID is the foundation for the magnetometer (Fig. 1). The thin-film SQUID is manufactured on the basis of unshunted $\mathrm{NbN}-\mathrm{NbN}_{x} \mathrm{O}_{y}-\mathrm{Nb} \mathrm{JJs}$ according to the technology presented in [6] and it has the following characteristics: $V_{g}=3.8 \div 4.0 \mathrm{mV}, R_{n}=15 \div 40$ $\mathrm{Ohm}, \mathrm{R}_{\mathrm{j}} / \mathrm{R}_{\mathrm{n}}==12 \div 44, \mathrm{I}_{\mathrm{c}}=3 \div 5 \mu \mathrm{A}$.

The RO magnetometer in the described computer-aided biomagnetic research systems is used in the mode of 2 nd order gradiometer, which makes possible the elimination of the noise whose sources are farther than the useful signal source. The component $d^{2} B / d^{2}$, i.e., the diagonal magnetic field gradient tensor component is recorded.

\section{MAGNETOCARDIOGRAPHIC INVESTIGATIONS}

Electrical heart activity is being recorded with the help of electrocardiograms (ECG) for almost 100 years. But the diagnosis of the heart activity state by ECGs is still an empiric process. Its potentialities can be considerably extended if, along with electrical activity, we will record the magnetic activity of the heart and use it for investigations violations of its electrical properties. For MCG recording a computer-aided system is developed. It incorporates: RO magnetometer whose detector is placed into the fiberglass cryostat, a PC that provides measuring process control and data collection/processing/ interpretation; a unit consisting of the electronic control and PC-communication components; a mechanical system providing the proper patient's and cryostat location. All the constructional elements are made of nonmagnetic materials, e.g., wood, textolit.

For recording MCGs, their initial processing and preparation of data required for interpretation of experimental data and medical diagnosis, three program packages are developed: input into a computer and preprocessing MCG; morphological analysis of MCG curves and contribution of instantaneous magnetic maps for different cardiocycle intervals; construction of electrodynamics heart models and evaluation of their parameters, localization of arrythmogenic activity sources.

\subsection{Signal processing}

When a single-channel magnetometer system is applied for the biomagnetic investigations oriented to a human heart, the observation points in this case are the intersection nodes located in the rectungular grid that is directed to the anatomical reference points of the patient's thorax. In our case, this is the $6 \times 6$ grid. When the measurements are finished and the data are preliminary processed, then the sets containing the magnetic field values present and the spatial grid nodes are at our disposal. It is necessary to supplement (smoothly) and interpolate the function of two variables at the points that are not the nodes. The approximation is performed by the 1,2,3-smooth piecewise polynomial functions or by the bicubic polynomials. When a function is supplemented, the search is performed and the equal-level (isolines) are drown. The PC-implemented algorithm permits to image the information as the coloured isolines or as the palette colour distribution when the colour saturation is determined by the magnetic heart field value.

A very important stage in the investigations aimed at heart examination consists in biomagnetic source localization. It is essentially the inverse magnetostatic problem since the biomagnetic signal frequencies are close to zero. A heart excitement source is simulated as a magnetic dipole. The software makes it possible to image the magnetic source moment vector position on a PC screen and also to make up a cardiocycletime source movement trajectory.

\subsection{Averaging algorithm under cardiac rhythm violations}

The averaging algorithm was applied in order to draw instantaneous magnetic maps. The semantical description was taken into consideration. These maps reflect the ventricle depolarization process for the 
case with the patients who suffer from polytop polymorphic extrasystole. When the algorithm was applied, there appeared the possibility to separate the extrasystoles of different localization in a proper way. Therefore, since it is possible to divide the processes running in myocardium when one rhythm type is changed into another, then it becomes also possible to determine the electrophysiological cardiac state with a higher accuracy and to assess the adequacy possessed by the therapy methods that are applied.

\subsection{Repolarization abnormality. Risk analysis. Smothness criterion.}

It is known, that the action potential duration dispersion is lower than the transmural difference between the refractor periods and it is also known that repolarization heterogeneities take place inside the very small domains on the whole ventricle surface. It is easier to detect these local tangential currents caused by small-domain repolarization non-homogeneity in MCGs than in ECGs. It is possible to reveal the initial myocardium pathology stages when repolarization non-homogeneity is present. The MCG ST-segment risk-analysis methodology suggests that four sequential magnetic field maps (when the first of them is the ST-segment begining and all the next-coming maps are taken under the $25 \mathrm{~ms}$ step) must be compared and that the so-called RS-score [7], i.e. the smoothness coefficient which is the sum of the coefficients reflecting a correlation between all the sequential maps and can assume the values from 0 to 3 should be calculated. The lower the numerical coefficient value, the lower is the map-form similarity degree and, therefore, the repolarization non-homogeneity and arrhythmia occurence risk are higher. When the norm/ pathology difference must be stated, the coefficient threshold is 2,3-2,5.

A set of various algorithms for MCG signal processing is used to perform a number of diagnostic examinations. For example, to investigate distinguishing features of electrophysiological processes in patients with cardiac rhythm disturbances an algorithm is developed for estimating repolarization homogeneity with the help of the magnetic field distribution smothness criterion suggested by the authors.

The methods applied for MCG data processing are as follows: MCG curve morphology analysis; visual analysis of instantaneous magnetic field distribution maps performed during a cardiac cycle; digital and statistical processing of magnetodynamic maps oriented to risk analysis; arrhythmogenic activity source localization. All these methods are aimed at cardiovascular system state examination.

\subsection{Diagnosis of iron overloading made magnetic mapping}

Let us represent the preliminary results reflecting the estimation performed for two groups of patients with respect to the extra iron storage: control team (cardiac disease case record is absent, 10 persons) and a welders team (10 persons whose labour service lengths are different). The greatest differences were detected in the magnetic maps at the points located in the left down grid quadrant wich corresponds to the left-ventricle projection. The field illustration for the ST interval is essentially changed in the case with the patients who has aquired extra iron and, probably, this circumstance emerged due to the fact that the magnetoplethysmogram registration was also performed along with a usual MCG registration. This magnetoplethysmogram registers the changes in the amounts of the blood containing extra iron which take place in the Earth magnetic field (EMF) during a cardiac cycle. The similar curves were also registered by us for healthy patients but it was done in the external magnetic field that was larger than the EMF. When a blood state is normal, this blood is a diamagnetic and during a cardiac cycle in EMF it creates little susceptibility variations which accordance to magnetic field of few pT. When the ferro-magnetic inclusions take place, then this effect is increased approximately by three orders and it is thus possible to observe it.

The iron overloading level can be estimated pursuant to the difference that is between the MCG curve form and the normal form if various comparison criteria are used. It is also very useful to apply the magnetometric methods in order to observe the dynamics of the uptake processes and of the processes of iron accumulation in different human body organs. 


\section{MAGNETIC SUSCEPTIBILITY OF HUMAN BLOOD AND INTERNAL ORGANS}

During the last years much attention is paid to the biological effects that occur when the excessive or deficient iron storage is observed in a human organism. A liver contains $60 \%$ of ferritin which is the protein. If the liver iron level deviates in one or another way, then such circumstance is very important from the clinical point of view. However, the existing methods that allow to determine this level are in close relation with biopsy (i.e. tissue damage) and, therefore, their wide application is impossible. But the problem can be resolved if the noninvasive biomagnetic measurements are applied as the basis.

\subsection{Method of investigation}

From a physical viewpoint the basis of measurement of iron concentration in liver consists of the presence of iron accumulation in liver by a paramagnetic complex - hemosiderin - with the magnetic susceptibility $\chi_{\mathrm{g}}=1600 \cdot 10^{-6}(\mathrm{SI})$. This susceptibility has an essential difference compared to the susceptibility of human body tissues, which equals about the susceptibility of water $\chi_{\mathrm{W}}=-9 \cdot 10^{-6}$. In the case of the influence of a test magnetic field a signal (which is proportional to the difference between the magnetic susceptibilitis of water and liver) can be recorded.

Two alternatives consisting of the utilization of alternating, or constant test magnetic field exist in the process of designing the measuring system. We have used the system with the alternating magnetic field becouse of its relatively low level. The biomagnetic system has a patient's bed which permits to move a patient in a vertical direction. The test magnetic field is created by the system of square Helmholtz coils which provide the alternating $(14 \mathrm{~Hz})$ test magnetic field of about $78 \mu \mathrm{T}$. The equality of the currents in the coils provides heterogeneity of magnetic field at least than $10^{-5}$ within $70 \times 70 \times 120 \mathrm{~mm}$ volume. A distilled water rubber bag is fastened to the cryostat tail and this is one for a parasitic paramagnetic air signal to be removed. Under the movement of a patients upwards the volume of the bag is decreased and the water is poured into expanding reservoir. The board for allocating the patient in the position "liver up" is fastened at the angle of $45^{\circ}$. The movement of the bed is brought about by the pneumatic driver. The bed distance is registered by a position transducer.

A measuring procedure is as follows. A patient idles (on a bed) at an angle of $45^{\circ}$. The bed is lifted in an upper position, then the bag fits tightly the body of the patient. Under measuring the bed is lowered to a distance of $5 \mathrm{~cm}$ for a time of $10 \mathrm{~s}$. The output signal of the magnetometer, which is proportional to the magnetic susceptibility of the liver, is input of a lock-in amplifier. A signal, which is proportionate to a distance between the liver and a bottom coil of the gradientometer, is registered from an output of a transducer of bed displacement. These signals are input of a two-channel chart recoder. Futher, the mentioned signals are sent conveyed to $\mathrm{PC}$ via ADC..

The calibration has been carried out by glass which has been filled up by a solution of $\mathrm{FeCl}_{3} \cdot 6 \mathrm{H}_{2} \mathrm{O}$ with equivalent concentration of iron of $0.1 ; 0.5 ; 1$ and $5 \mathrm{mg} / \mathrm{ml}$. Here it was enough to measure two values of the concentration, since both the theoretical calculations [8] and experimental results show a good linear relation between the output signal and the concentration of iron.It is seen from Fig. $2 a$ that the iron concentration higher than $0.1 \mathrm{mg} / \mathrm{g}$ liver can be measured by the described measuring system. Fig. $2 \mathrm{~b}$ presents the results of the measure-ments of the magnetic susceptibility of the liver of both a healthy man and a welder suffering from occupational diseases. The iron concentration for a group of healthy subjects varies within $0.5-0.8 \mathrm{mg} / \mathrm{g}$. A high iron concentration about $3 \mathrm{mg} / \mathrm{g}$ has been registered for the welder. The distance to object is measured relative to its upper position.

\subsection{Magnetoplethysmography}

(MPG) means the measurement of the change of the blood volume in human organs for a period of cardiocycle by a signal from blood in external test magnetic field. This signal can be elicited due to a 


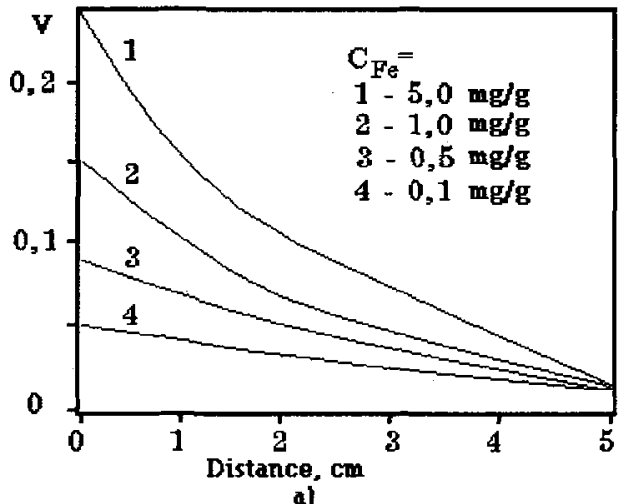

a)

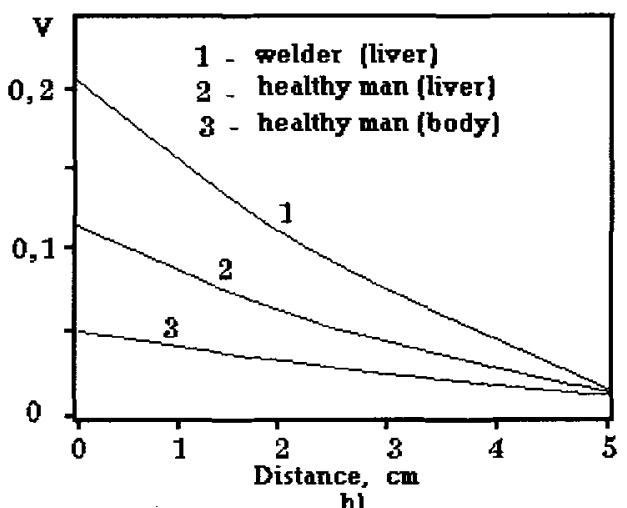

b)

Figure 2: The dependence of the output signal of biosusceptometer on a distance to the set of the rubber capacities with different calibration values of iron concentration (a), the liver of a healthy man and welder (b).

difference between the magnetic susceptibilities of blood $\chi_{b}=5 \cdot 10^{-6}$ and body. The essence of the method of MPG developed in [9] based on the application of a DC test magnetic field The measurement is performed by means of the same magnetocardiograph since both MPG and MCG signals are input signals of the system. The MPG signal is received as a difference of MCG + MPG signals for opposite directions of magnetic field. However, under the measurement of MPG by a constant field the heart should work for the permanent conditions that is difficult to realize in practice. We use the AC field with a frequency of $114 \mathrm{~Hz}$ for receiving the MPG, then both the noise of the magnetometer and various external noise are considerably decreased. Furthermore, a "parasitic" MCG signal has no influence for this frequency. The blood iron content level is so high among some welders that it became possible for us to have registered liver MPG for the first time. Thus the system allows to elicit an enhanced concentration of iron in blood and liver of patient. Consequently, this system can be used for a diagnosis of a few diseases: hemachromatosis, hemosiderosis, thalassemia, and poiso-ning of iron complexes. The magnetic biopsy of liver has been complied and the MPG of heart and liver have been received. The groups of healthy subjects and patients with different pathologies have been examined.

\section{References}

[1] Gutmann P. Electr. Letters 15 (1979) 372-373.

[2] Drung D. Supercond. Sci. Technol. 4 (1991) 377-385.

[3] Sosnitsky V.N., Vojtovich I.D. Control system and mashines (USIM), Kiev. 3 (1995) 31-46.

[4] Vojtovich I.D., Sosnitsky V.N. et al. "Pulse magnetometer", XIII Int. Symp. Low Temp. Phys. and Cryoelectronics, Jena, Germany (1981) pp. 203-207.

[5] Muck M., Rogalla H., Heiden C. Appl. Physics A46 (1988) 97 - 101.

[6] Krivoj G.S., Komashko V.A. Mod. Phys. Lett. B5 (1991) 365-373.

[7] Makijarvi M."MCG. and risk analysis" 8th Int.Conf. Biomagnetism, Munster (1991) 523-530.

[8] Romanovich S., Sosnitsky V., Vojtovich I. "Investigation of biomagnetic field using two-dimensional model of secondary sources " 9th Int. Conf. Biomagnetism, Vienna 1993, Biomagnetism: Fundamental Research and Clinical Applications, C. Baumgartner et al.(Eds.), Elsevier Science, Amsterdam, 1995, pp. 814-818.

[9] R. Maniewski R., Katila T., Poutanen T. et al., IEEET BME-35 (1988) 662-670. 